\title{
VIRTUAL ENTERPRISE NETWORK SOLUTIONS TO SUPPORT E-LEARNING SITES DEVELOPMENT
}

\author{
Rosu, S. M.; DRAGOI, G.; Rosu, L. \& GURAN, M.
}

Abstract: Informational society construction can't be realized without research and investment project in IT\&C and education domain. The rapid development of a new technologies lead to invariable apparition of new evolving products on the market. For to keep pace with competitor companies it's imperative than the enterprises to assure a permanent training their employees adapted to market changes or trends and enterprise specifically. This work analyse the network architecture for a virtual enterprise geographic dispersed as support for virtual project development by virtual teams work. Therefore, to knowledge dissemination at each enterprise level, this paper provided a model for a three layers e-learning site built in the enterprises using open source software (OSS), starting from object modeling technique (OMT).

Key words: client-server architecture, collaborative work, e-learning site, virtual enterprise network, virtual teams
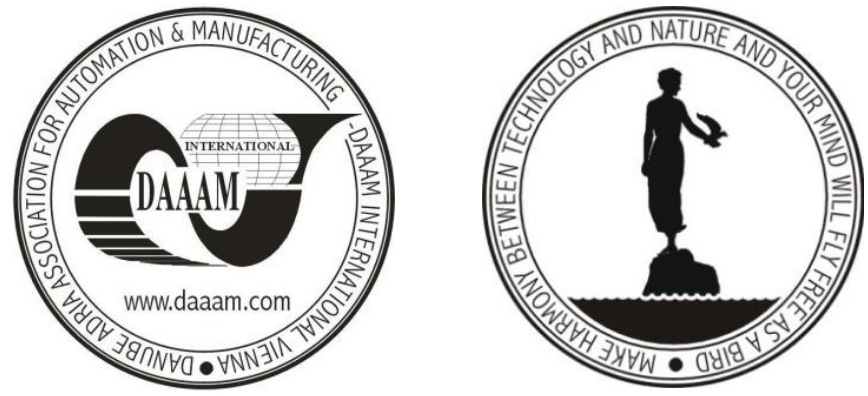

Authors' data: PhD. Student Rosu, S[ebastian] M[arius]*; Prof. Dragoi, G[eorge]**; M.Sc. Rosu, L[uminita]***; Prof. Guran, M[arius]**, *Special Telecommunications Service, 323A Splaiul Independentei, Sector 6, 060044, Bucharest, RO, **UPB, FILS, PREMINV Research Center, 313 Splaiul Independentei, Sector 6, 060042, Bucharest, RO, *** UPB, PREMINV Research Center, 313 Splaiul Independentei, Sector 6, 060042, Bucharest, RO, sebastianrosu@stsnet.ro, gdragoi@mix.mmi.pub.ro, luminita.rosu@yahoo.com, mguran@mix.mmi.pub.ro

This Publication has to be referred as: Rosu, S[ebastian] M[arius]; Dragoi, G[eorge]; Rosu, L[uminita] \& Guran, M[arius] (2010). Virtual Enterprise Network Solutions to Support E-learning Sites Development, Chapter 63 in DAAAM International Scientific Book 2010, pp. 725-742, B. Katalinic (Ed.), Published by DAAAM International, ISBN 978-3-901509-74-2, ISSN 1726-9687, Vienna, Austria DOI: $10.2507 /$ daaam.scibook.2010.63 


\section{Introduction}

In the $21^{\text {st }}$ century, the enterprises continuous implement IT strategies \& architectures to improve manufacture, research, products quality, sales and services and to costs control. All organizations use today Intranet or Internet technologies to attract, retain and cultivate relationships with customers, streamline supply-chain, manufacturing, procurement systems and automate corporate processes to deliver the right products and services to customers quickly and cost-effectively, also to capture, explore, analyze, and automate enterprise processes information on customers and company operations in order to provide better business decisions (Dragoi et al., 2006). For business, e-service is going to be a new way to save money, to revenue growth, and faster development model. For end-users, e-services increase productivity and simplify life, take advantage of more sophisticated and specialized services on as needed basis. At the level of production-dedicated enterprises, eservices are (see figure 1): business-to-business (B2B), intra-business (1B), and business-to-customer (B2C), (Dragoi et al., 2007). New enterprise model architecture uses the Intranet/Internet/Extranet infrastructure and technologies.

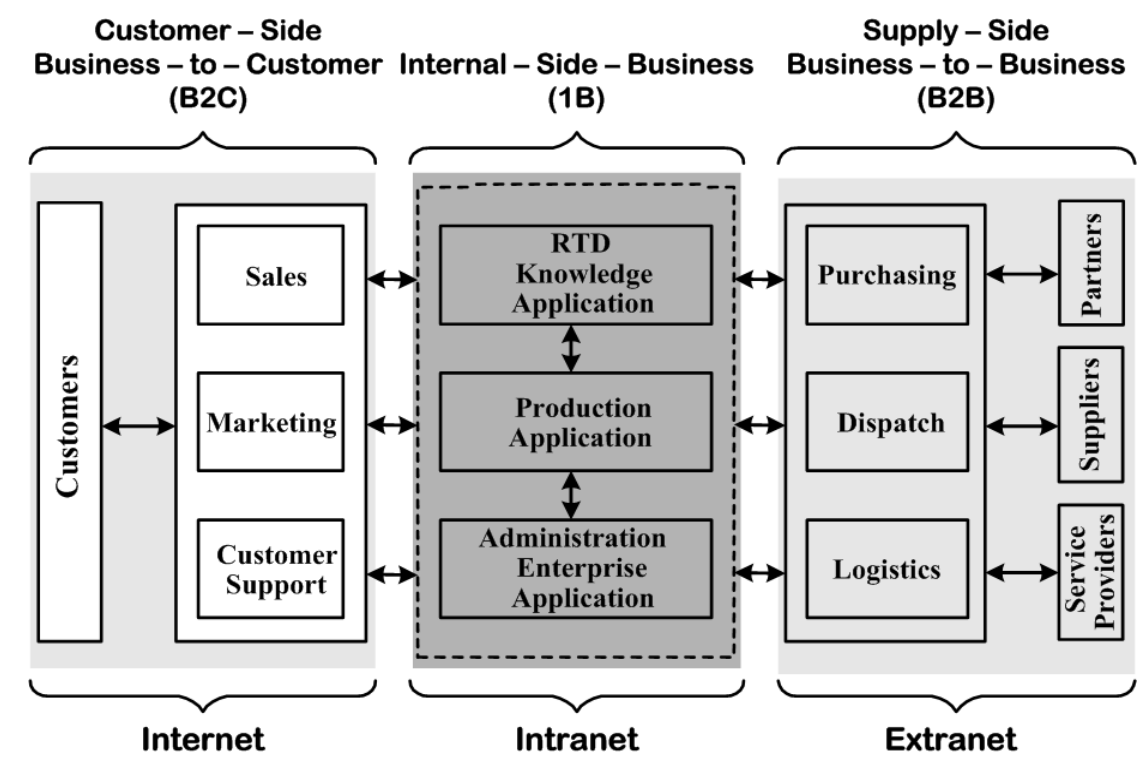

Fig. 1. The exchange of information services and goods through networks

E-learning is an efficient method to put at employees disposal needed the knowledge and information's for to keep pace with quick market changes and furnish the possibility to check their assimilated knowledge level. E-learning begins to be use in the training departments of companies, schools and universities like an alternative or complementary to traditional training methods. This training is process assist by Internet or by means of Internet/Intranet/Extranet platform.. By e-learning it is created for employees a possibility to exploit the existing knowledge resources by knowledge presentation in a more accessible format, individualized and pointed to training beneficiary. Nowadays, the enterprises manage the professionals reconversion for their people based on e-learning products or start to development properly e-learning trainings systems. 


\section{The collaborative virtual teams work in virtual enterprise network}

The term virtual enterprise (VE) has been used in articulating the strategy for the $21^{\text {st }}$ century global manufacturing enterprises. One of the key requirements is to develop an Information System infrastructure to integrate and control the interoperability of the distributed, heterogeneous and concurrent systems in the participating organizations.

As a general requirement for an infrastructure support is than the enterprises must be able to inter-operate and exchange information's and knowledge in real time so that they can work as a single integrated unit, although keeping their independence/autonomy. For the future, e-services and e-business, as were defined, require the enterprise re-thinking and re-modeling, with the system and applications design for an efficient use of new network technologies. The perspectives of this kind of manufacturing and economy, named shortly new digital economy, we can see the product perspective (holistic product view, product life-cycle, value-network integration, etc.), business organizational perspective (new organizational form, customers and suppliers integration, collaborating organization etc.), the technology perspective (technological building blocks, infrastructures, interoperability etc.) and the individual perspective (skills, workspaces, collaborating individual, different rolls: worker, consumer, citizen), (Rosu et al., a2008).

The range of software tools and technologies available to support collaborative design activities is growing dramatically. The deployment of these tools and technologies opens up the possibility for establishing virtual design offices that bring together people from the entire design network: making the right decisions at the right time enabled by the right conversations supported by the right tools and information. However, the vision, skills and competencies needed to exploit such technologies are cross, disciplinary and the critical mass needed to exploit such tools is not readily accessible within many organizations. In addition, the resource cost of using these tools and technologies to support designers carrying out distributed design processes is often significantly greater than the cost of acquisition. It is not surprising that the potential offered by the research into and creation of collaborative tools remains untapped. Each of the virtual design offices was created using only commercially available software and hardware.

Virtual teams for engineering design are becoming more commonly used in industry and the engineering education community must prepare graduates to be employed in such work environments. It is inevitable that multidisciplinary teams for product design, with members located in different geographic locations, will become more commonplace in the future. It is widely understood that successful design is often a highly collaborative team based activity (Dragoi et al., 2007). To be effective, a virtual team must be able to communicate, collaborate and coordinate, all at distance. Though some corporation are practicing a form of distributed design, a documented procedure for conducting distributed design and product development has yet to be created, tested and distributed.

Enterprises are now facing growing global competition and the continual success in the marketplace depends very much on how efficient and effective the 
companies are able to respond to customer demands. The formation of virtual enterprise network is taking up momentum to meet this challenge.

The idea of virtual enterprise network (VEN) is meant to establish a dynamic organization by the synergetic combination of dissimilar companies with different core competencies, thereby forming a best of everything consortium to perform a given business project to achieve maximum degree of customer satisfaction. In this emerging business model of VEN, the decision support functionality, which addresses the issues such as selection of business partners, coordination in the distribution of production processes and the prediction of production problems, is an important domain to be studied.

A virtual product development by the virtual teams in a VE is a temporary alliance of teams that come together to share skills, abilities and resources in order to attend a project opportunity and whose cooperation is supported by computer network and adequate tools, competencies and special application software. VE operates as nodes in a network. A different architecture, engineer and construction organization, a fresh virtual team is needed every time for every new project. Innovative techniques to co-ordinate and manage information, resources and documents need to be developed to integrate successfully and reduce lead times, increase quality and keep within budget constraints. Consequently, the partners in the VE need to exchange legacy data and migrate with other systems outside their own secure corporate boundary.

In order to achieve collaboration between different actors in the VE, there needs to be common processes supporting the distributed product development process. In this context a VEN is a way for businesses to achieve virtual scale enabling them to operate as if they had more resources and capacity than they actually have. A Virtual Enterprise Network (see figure 2) needs its own Private Member Collaboration System to communicate and develop its projects and bids.

\section{Enterprise 1}

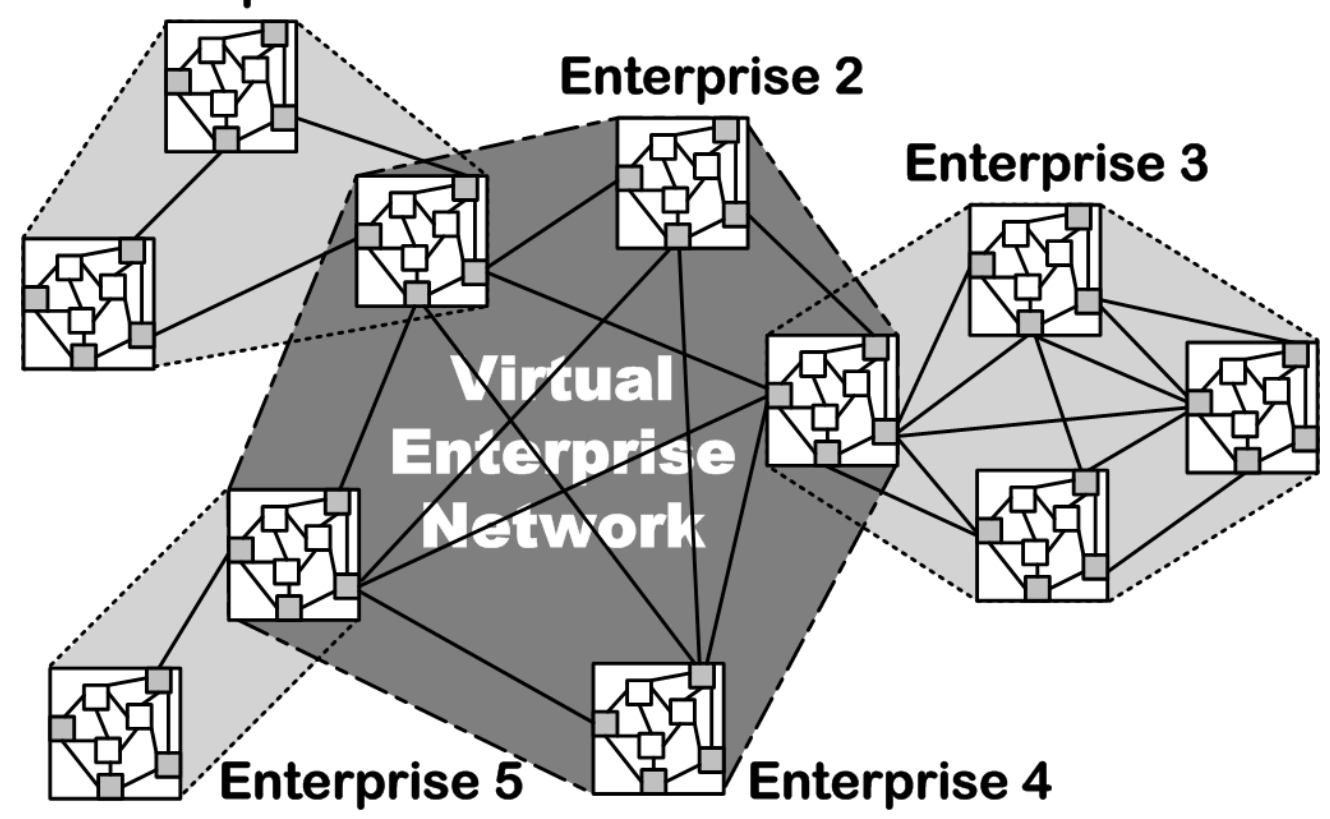

Fig. 2. The cooperative enterprise projects in VEN 
What is a virtual enterprise network (VEN) solution?

- A way for businesses to achieve virtual scale enabling them to operate as if they had more resources and capacity than they actually have;

- Allowing them to operate with all the resources and reach of a large enterprise but without sacrificing their speed, agility and low overheads;

- Enabling them to compete for bigger more profitable contacts with higher innovation and design elements with bigger customers who are prepared to have strategic partnerships with their suppliers (Thuraisingham, 2003).

A Virtual Enterprise Network needs its own Private Member Collaboration System to communicate and develop its projects and bids. It needs its distinctive Network Business Applications such as Capability Aggregation and Tender Matching to enable it to function effectively as a co-operative in both pre-sales and contract operations. It also needs a Public Web Site to manage its interactions with potential customers and new members.

A hierarchical network design model breaks the complex problem of network design into smaller, more manageable problems. Each level, or tier, in the hierarchy addresses a different set of problems so that network hardware and software can be optimized to perform specific roles. Devices at the lowest tier of the hierarchy are designed to accept traffic into a network and then pass traffic up to the higher layers. The core of the network has one purpose: to provide an optimized and reliable transport structure by forwarding traffic at very high speeds. In other words, the core layer should switch packets as fast as possible.

Also, today, what do you want in an e-teams with multidisciplinary optimization? First, for your enterprise: low risk, low cost, single point of support; reap benefits as soon as possible, ready for unpredictable demand and growth, future capabilities. Secondly, for your customers: excellent response time, ability to build competitive advantages from a wide variety of applications (use specialized products for unique features, integrate them for seamless customer support etc), ensure optimal customer experience to build loyalty, customer trust (security features build trust: validate who is doing business with whom; secure financial transactions, protect internal assets, halt spread of viruses, protect against hackers), high availability, etc .

Innovative techniques to co-ordinate and manage information, resources and documents need to be developed to integrate successfully and reduce lead times, increase quality and keep within budget constraints. Consequently, the partners in the virtual enterprise need to exchange legacy data and migrate with other systems outside their own secure corporate boundary.

In order to achieve collaboration between different actors in the Virtual Enterprise, there needs to be common processes supporting the distributed product development process.

Virtual teams do not operate like traditional physical teams, as their requirements reflect a whole new way of communicating, working collaboratively, sharing information and mutually supporting other team members. The new technologies and approaches required to achieve this are completely alien to most of our present organizational culture. And this is why they fail. 
Rosu, S.M.; Dragoi, G.; Rosu, L. \& Guran, M.: Virtual Enterprise Network ...

Cooperative processes are not the automatic results of implementing collaborative, real-time communication technologies, but the result of a carefully designed and systematically maintained virtual team development plan.

We purpose a general architecture for a large enterprise or an industrial holding, geographically dispersed, in order to achieve collaboration between different actors in the virtual enterprise, there needs to be common processes supporting the distributed product development process (see figure 3 ).

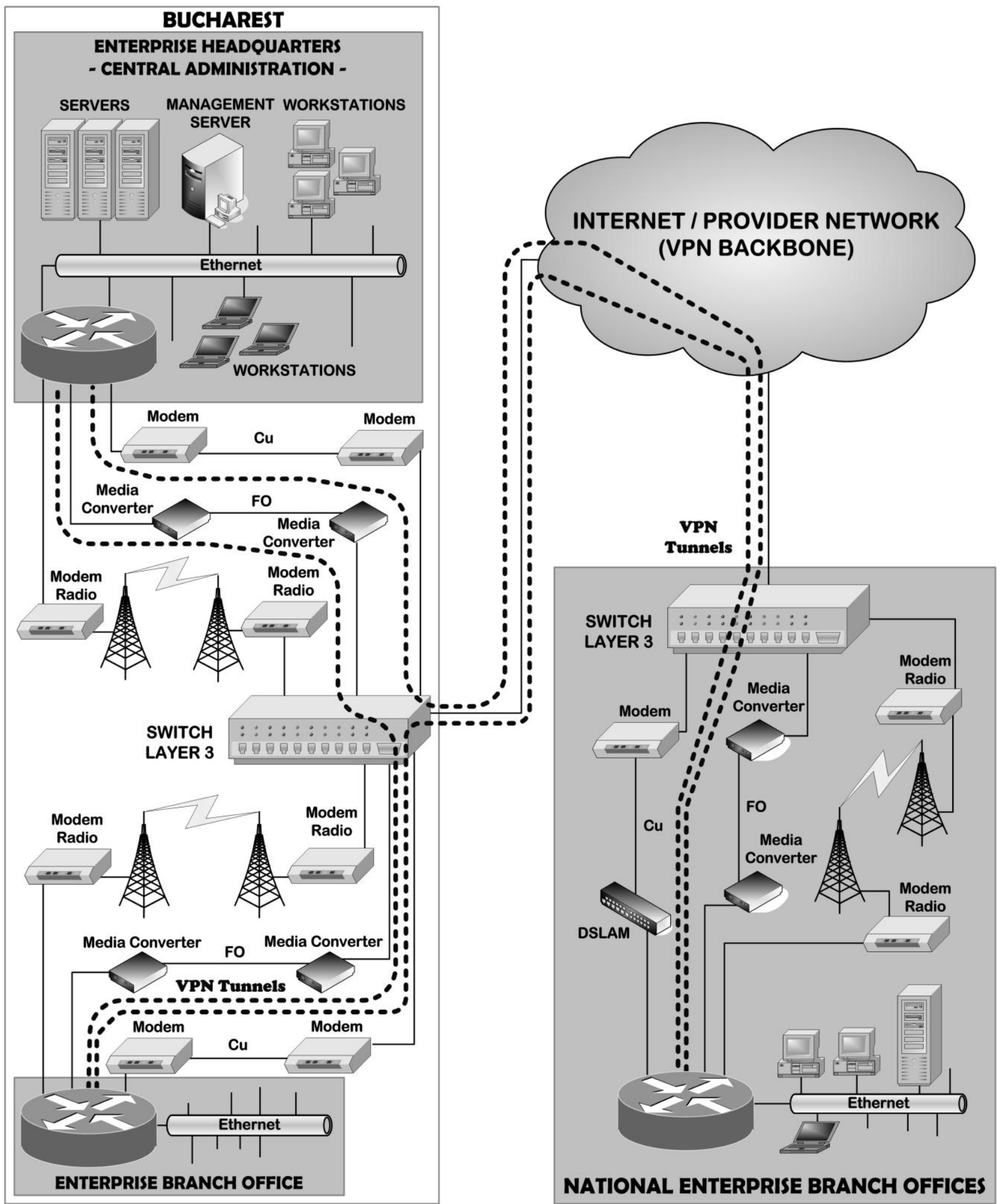

Fig. 3. A network solution with 3 loops for an enterprise geographic dispersed 
The Internet is a world-wide conglomerate of different networks that communicate among each other via a common protocol, independently of the hardware type used.

Various network services can be used by everyone, either supplying or demanding them. A large range of distribution, the platform independence, an big number of user friendly services that are easily accessible through the World Wide Web as well as the open standards used and free or budget-priced products (such as browsers, html editors, software updates) have lead to a high and continuously growing proliferation of the Internet (Shakya et al., 2008). The advantages offered by the e-platform for covering the information needs are held to be the following (Dragoi et al., 2007):

- Reduction of local barriers by means of world-wide information offers;

- Reduction of time barriers by means of permanently available information;

- Reduction of (transaction) costs by way of automation of information processing on the supply and/or the demand side;

- Improved coordination and cooperation with external partners using an integrated information and communication platform (e.g. platform independence, information exchange without media ruptures).

However, the application-to-application communication problem still exists. Businesses have needed a standardized way for applications to communicate with one another over networks; no matter how those applications were originally implemented (Ward \& Peppard, 2002).

Web Services, the latest evolutionary step in distributed computing, represent exactly this solution by providing a standardized method of communication by means of which different applications can be integrated together in ways not possible before. Different applications can be made to call on each others resources easily and reliably, and the different resources that applications already provide can be linked together to provide new sorts of resources and functionality. Moreover, the application integration becomes much more flexible because Web Services provide a form of communication that is not tied to any particular platform or programming language.

\section{Virtual enterprise network solutions}

Appearance of virtual networks is related to the evolution switches. A virtual network (see in figure 4 the Virtual LAN) is to combine a group of users regardless of their geographical position but such a manner that it flows together and to provide the best performance. The second advantage of a virtual network consists of administrative solutions which accompany the products, allowing users moving from one group to another through a simple reconfiguration of the equipment. An example of establishing a virtual network is presented in figure 5 .

Extension for virtual networks, more than Ethernet switches or token ring, the ATM network federating other techniques are required to convert cells in the frame for local network emulation on switched virtual circuits. Currently operational standard is LANE (LAN Emulation). 

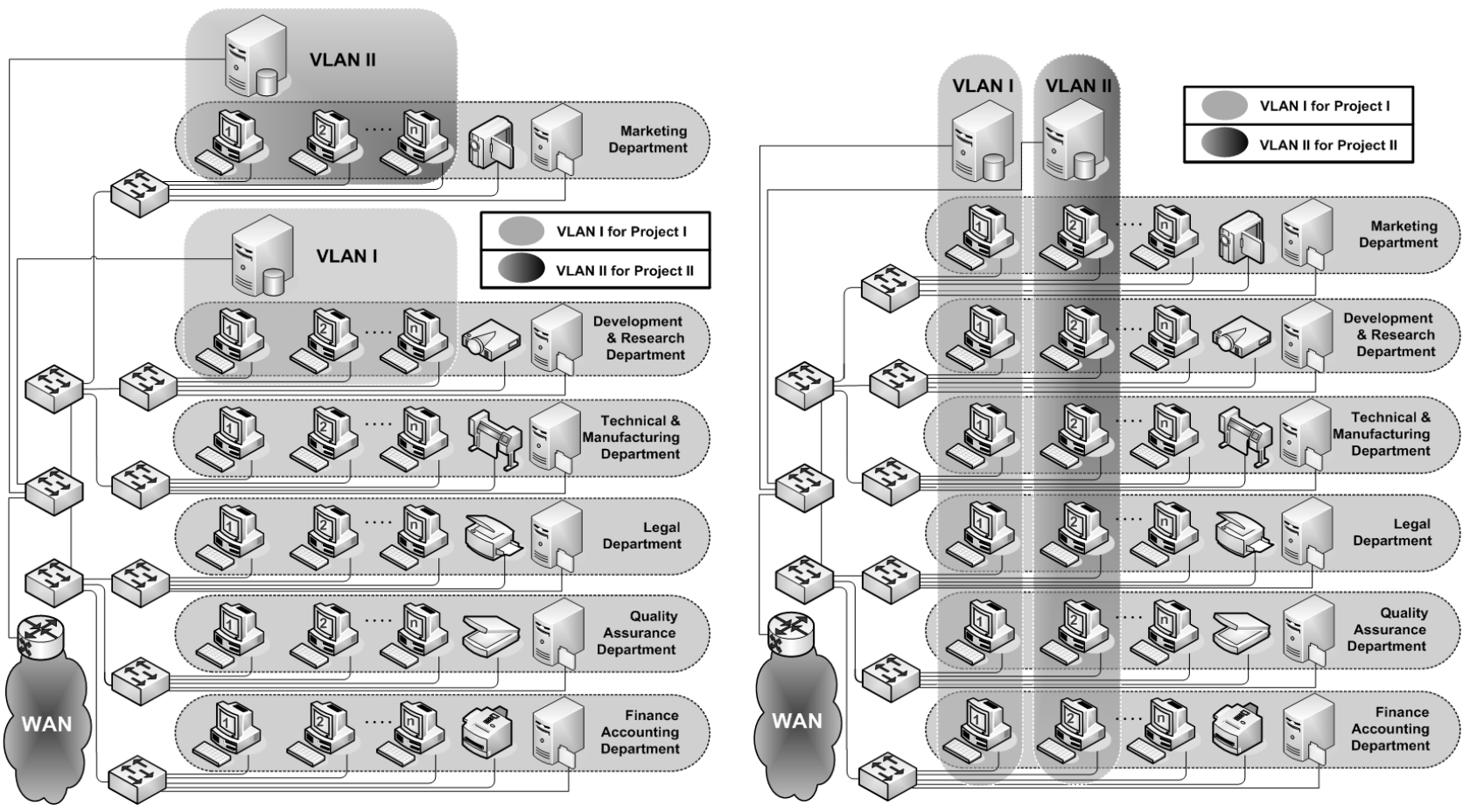

Fig. 4. The enterprise departmental and interdepartmental VLANs

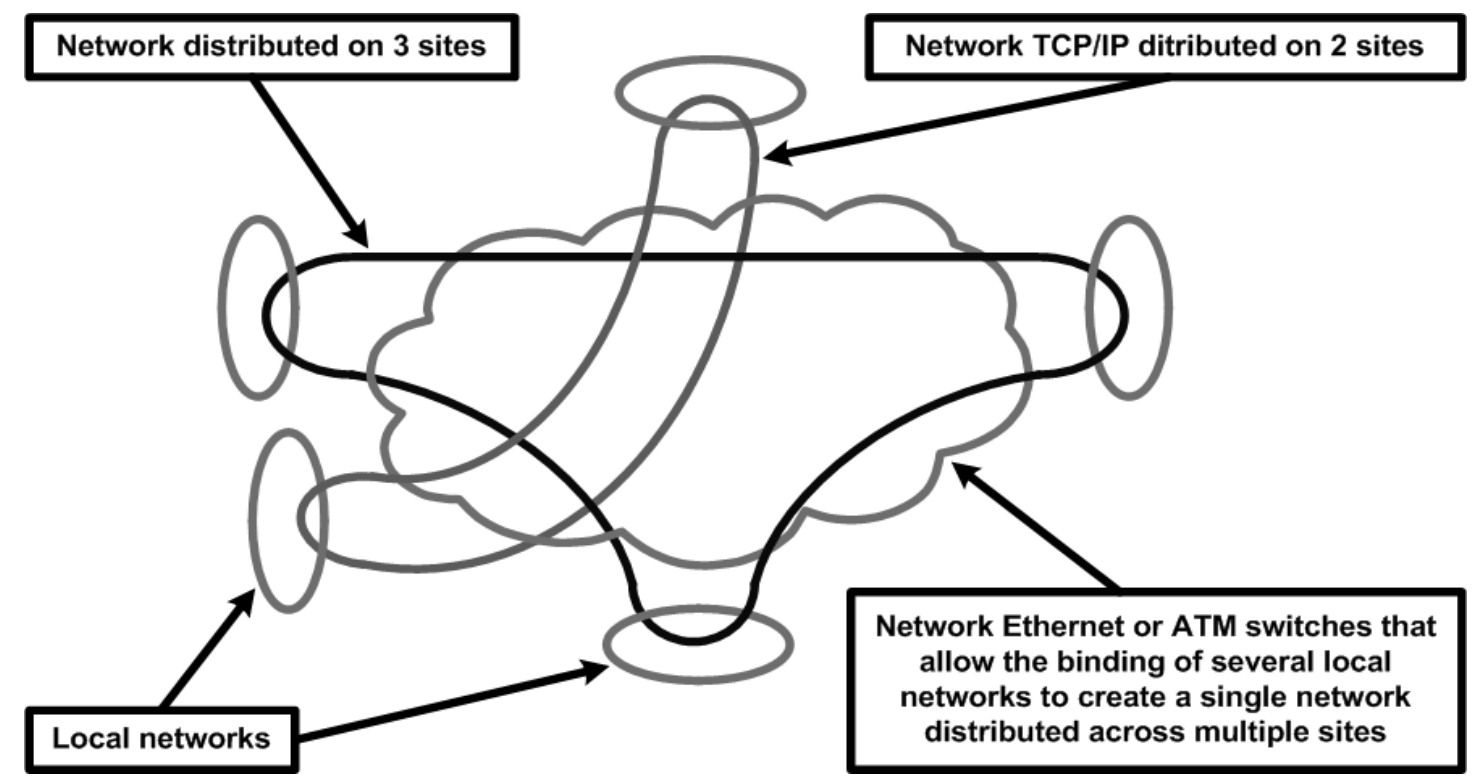

Fig. 5. Principle of virtual networks

Emulation of a local ATM network requires the use of virtual channels (Virtual Channel Connection) predefined or dynamically allocated. Virtual networks do not take account of geographical restrictions can be divided into different strength and vice versa, a concentrator can accommodate more VLAN.

Protocol LAN Emulation (LANE) allows virtual network level 2 - those that not only deals with MAC addresses. As with VLANs, various interconnection links ELAN pass through routers. It may be a position switch edge device, or pass directly through the ATM.

Virtual network interconnection can be achieved at Level 3 by standard MPOA (MultiProtocol Over ATM) whose principle is based on the notion of server paths and 
multi-switch (multi-layer edge devices). By definition MPC (MPOA Client) and MPS (MPOA Server) configuration parameters obtained from the LECS (LAN Emulation Configuration Server).

MPOA virtual networks are called IASG (Internet Address Summarization Groups) and may correspond, for example, IPX networks or IP subnetworks. Rule provides support OSPF and RIP protocols.

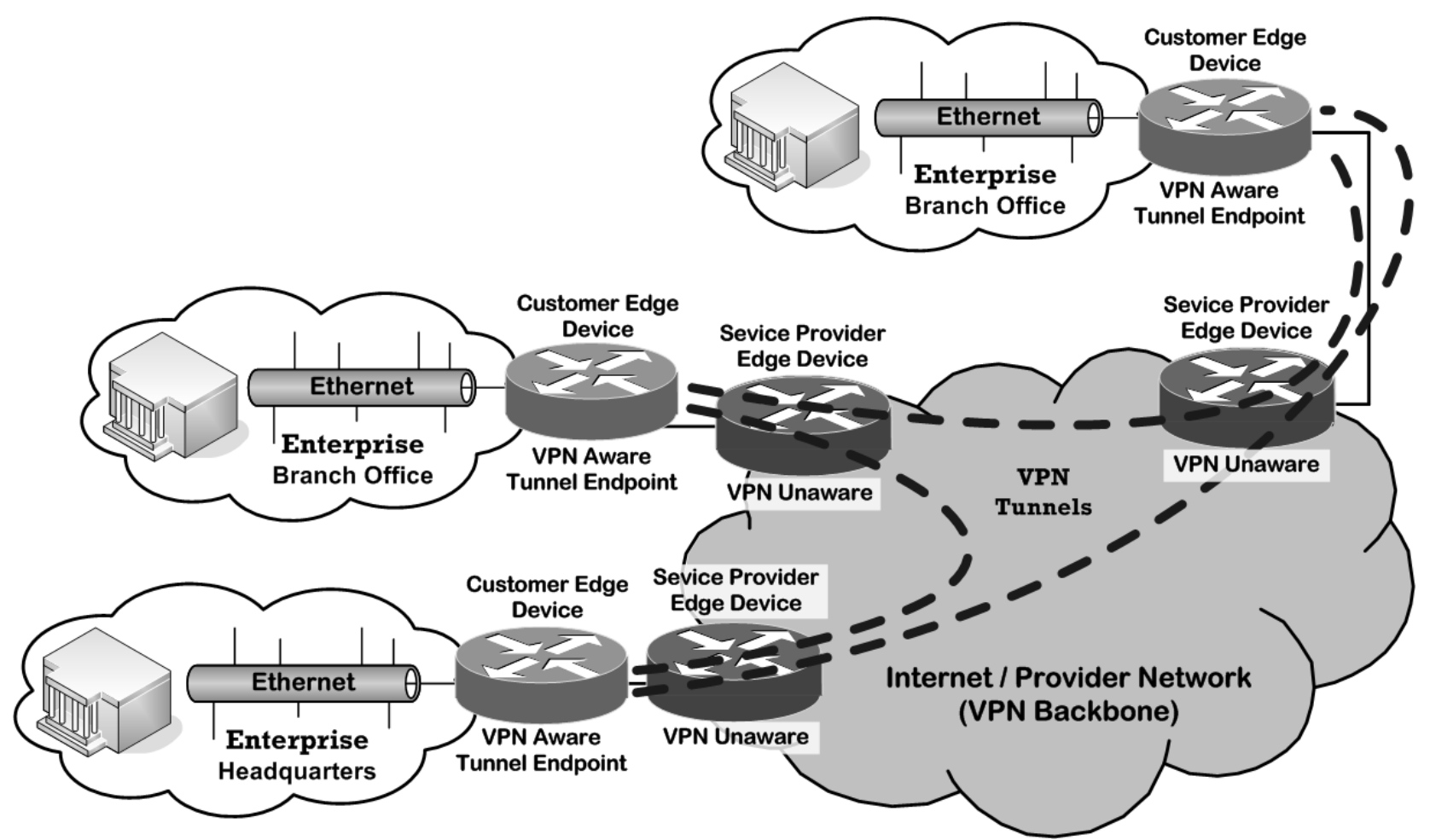

Fig. 6. Customer-Edge based site-to-site VPN general architecture
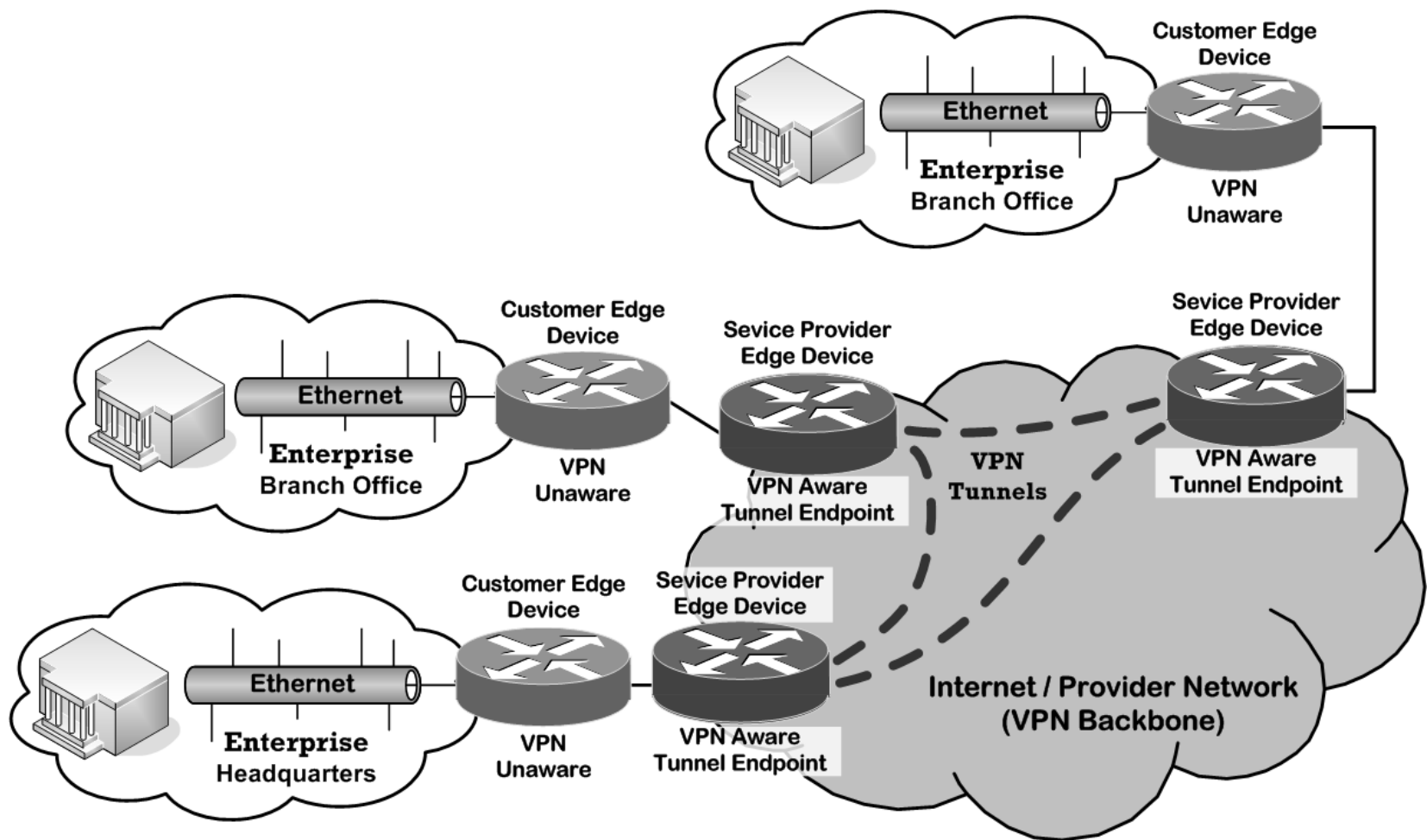

Fig. 7. Provider-Edge based site-to-site VPN general architecture 
LANE virtual network deal so level 2 and MPOA virtual network level 3 (routing), the specifications are set by ATM forum. In fact, the VPN (see figure 6 and 7) is a network emulated (the virtual) built on public infrastructure (shared), dedicated to a client (the private) to connect users in locations and to ensure similar conditions of integrity, confidentiality and quality similar with those of a private network. A virtual private network (VPN) allows the provisioning of private network services for an organization or organizations over a public or shared infrastructure such as the Internet or service provider backbone network. The shared service provider backbone network is known as the VPN backbone and is used to transport traffic for multiple VPNs, as well as possibly non-VPN traffic. VPNs provisioned using technologies such as Frame Relay and Asynchronous Transfer Mode (ATM) virtual circuits (VC) have been available for a long time, but over the past few years IP and IP/Multiprotocol Label Switching (MPLS) - based VPNs have become more and more popular (www.cisco.com).

We propose that a possible solution for an enterprise geographically dispersed following structure VPN to create a VE: a three layers network organized on a provider network (see figure 8). We defined the 3 layers (access, aggregation and transport) taking into account the territorial expansion of VPNs: local, metropolitan (county) and national (international, inter-networks, extranet) VPNs.

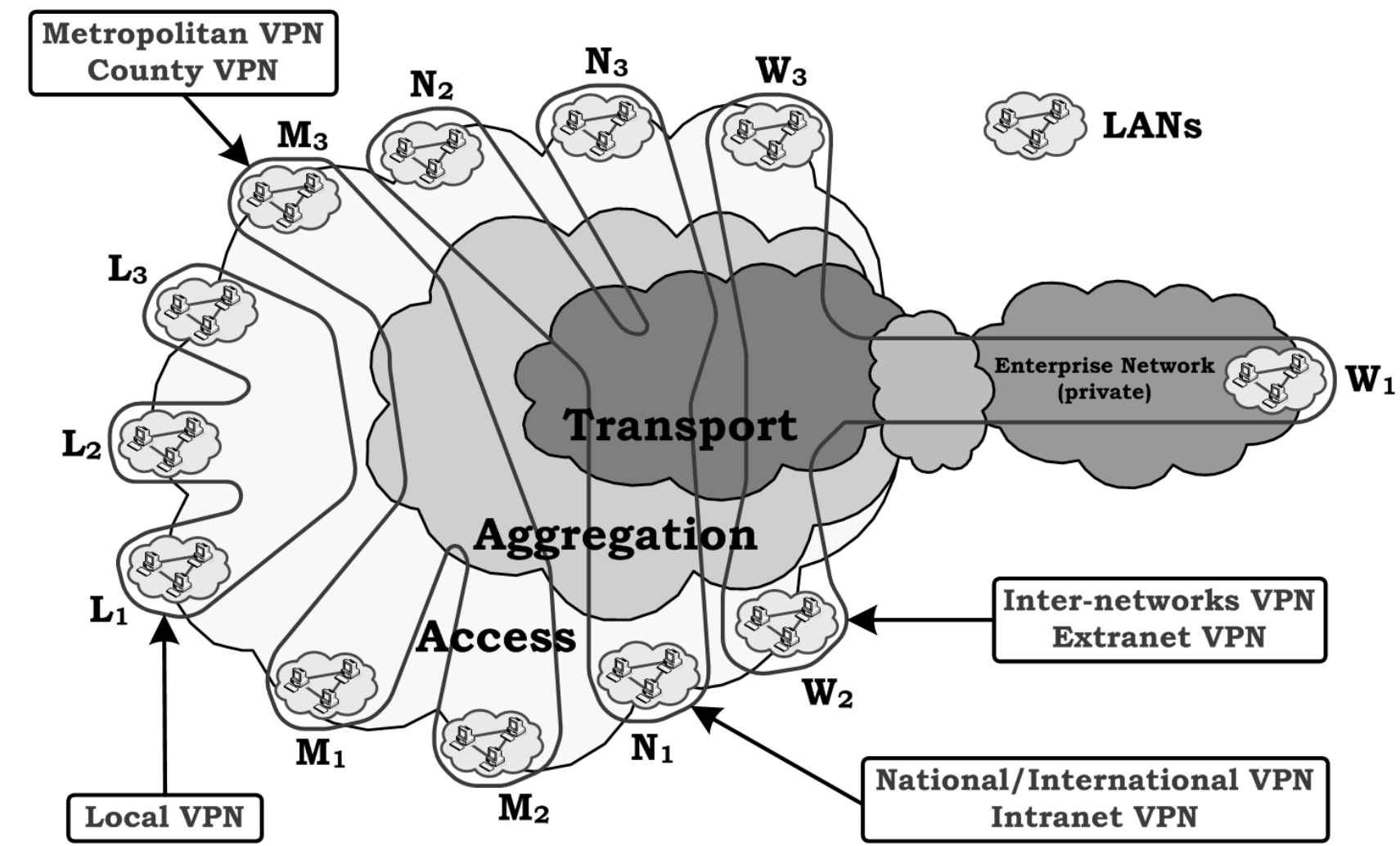

Fig. 8. VPNs possible structures on a provider network

Clear trend is now evolving to intranets and extraneturi defined logic, which will lead to the reintegration of the various networks in a single logical subdivisions with no physical. Structures that allow the approximation of this goal are virtual private networks. Newer, VPNs can be used in different ways to support business processes, is the ideal solution if it is not efficient in terms of construction costs of a particular 
network for a firm with a workforce highly mobile, or for small firms that can not justify the cost of their telecommunications network. VPNs can be purchased from a telecommunications company and as an alternative they can create by using existing network infrastructure as the Internet or public switched telephone network, and software through the tunnel crossing.

\section{A three layers e-learning site architecture}

New information technologies and communications change the perspective about educational practice integrating the educational process with modern learning methodology specific to information society (see figure 9). E-learning is not ready to replace traditional education systems, but aims to strengthen the learning process. Across organizations, the e-learning can promote collaborative learning, optimizing the process of organizational learning specifically modern enterprises. A system of eLearning (training distance or virtual education) is a planned experience of teachinglearning, organized by an institution which provides the material in a sequential and logical order to be assimilated by students in fashion own, without work to constrain the co-presence or synchronization.

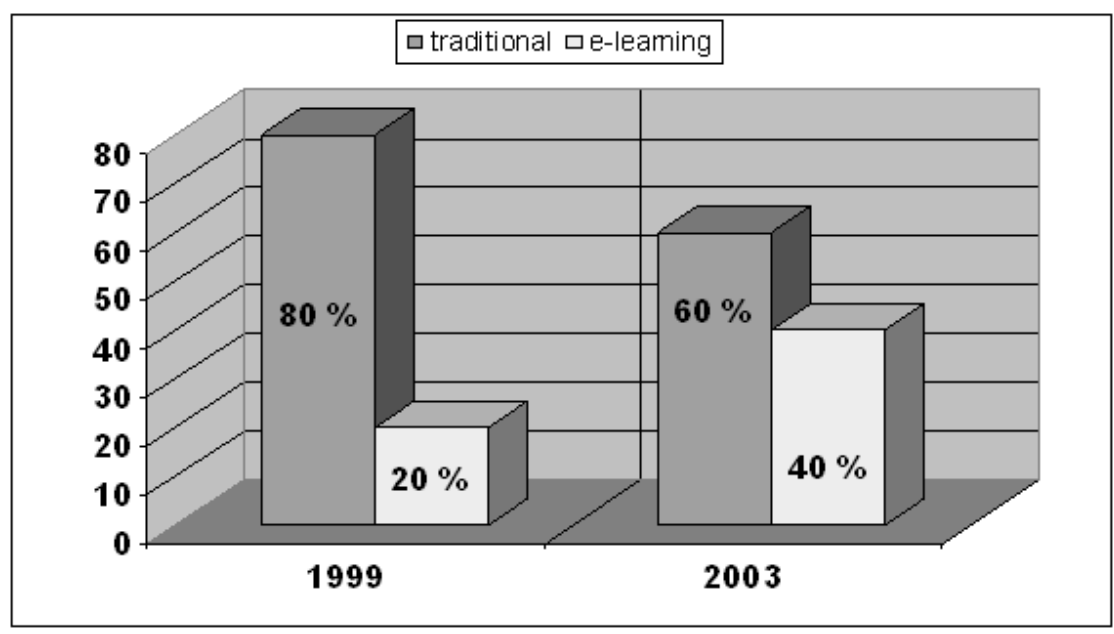

Fig. 9. Traditional education vs. e-learning

E-learning sites can be developed using OMT (Object Modeling Technique) methodology - methodology of analysis and object-oriented design. An object can be seen as an entity that incorporates both data structures, called attributes, and behavior, called operation. An object must have the following features:

- Identity - the object is a discrete entity, which is distinguished from other entities (e.g. a window on a workstation, an isosceles triangle, a list of people).

- Classification - objects with the same attributes and operations are grouped into classes; each object with the same attributes and operations can be considered as an instance of a class (e.g. window, triangle, list).

- Polymorphism - the same operation (with the same name) may have different behavior in different classes (e.g. move a window, move a triangle); operation 
move means different things depending on the subject on which it applies concrete implementation of an operation in a class is called method.

- Inheritance - the attributes and operations are transmitted of all downward classes in a hierarchical relationship.

In building the model objects the most important stage is the evidence that objects with their attributes and operations, and relations between them. Concepts used in this model are classes and objects, diagrams, attributes, operations and methods. Object model is the most important stage of the OMT methodology because it shows the static structure of real system. Model objects are represented using graphs of objects that are graphs whose nodes are objects and whose arcs represent relationships between objects. Steps in obtaining the objects model are: identifying candidate classes, classes keeping accurate, the preparation of a dictionary, the identification of associations, associations keeping accurate, identifying attributes, attributes keeping accurate, refine the model and grouping classes into modules.

The concepts used in implementing the model objects are: the class concept, the object concept, the attribute concept, the diagram concept, the operation concept, method, links and associations. An object is an abstract concept and it is built for problems understanding before solution implementation. Generally, an object is an entity that can be distinguished among other entities and have significance in modeling application context. Each object has one specific identity even if it is similar to other objects. An object class describes a multitude of objects with similar properties, similar behaviors and similar relations regard others objects.

In the modeling of objects held the following:

- Web page has been modeled as object (by means the item page will have the general characteristics of all elements contained in the hierarchy - these features will be: background, fonts, artistic effects, buttons navigation, etc.);

- Collection of Web pages with the same attributes will shape the class of objects.

For a site designing, OMT method can be particularized by us as well as figure 10 (Rosu et al., b2008). For this site there were identified and created the following classes: index, main_page, main_theory, main_solving_problems, theory_pages, etc.

After that, there were identified associations between objects, relations, attributes and there was created the database. The database development using this model requests an application server and a database server. Application servers are responsible for communications between users. Usual steps in users working process with databases are the following:

- The user makes a request for certain dates (e.g. a special form, a rapport or a situation);

- The application server receives the request, locates the files and gives request to PHP for processing;

- The PHP starts processing, makes the connection with database and sends the request;

- The Database server receives the request, processing and sent to PHP the result;

- PHP returns the result to application server;

- Server applications send the result to the user. 


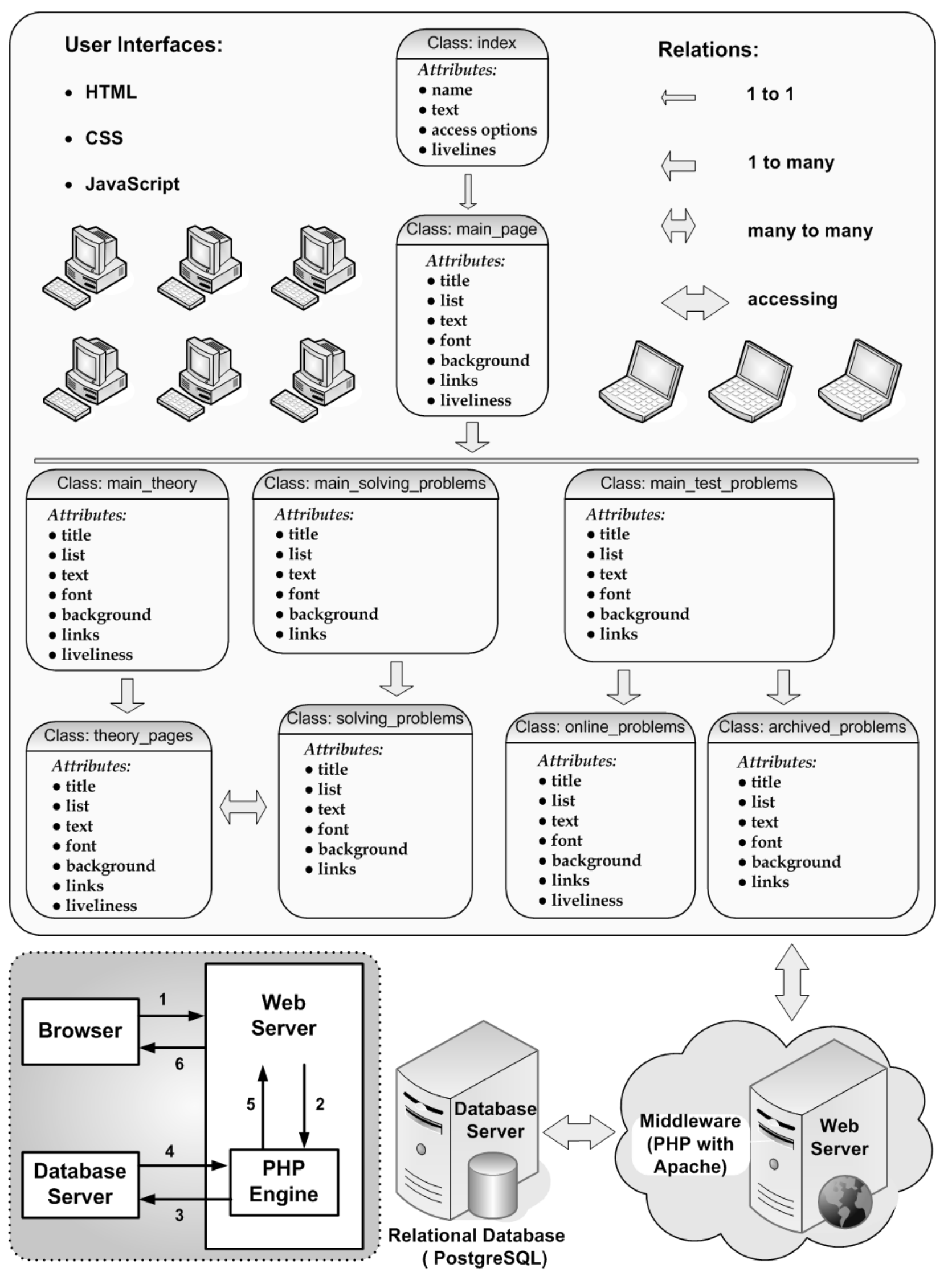

Fig. 10. An e-learning site architecture using OMT methodology

Here, we used a 3 level Client-Server architecture (see figure 11):

- Database server represents the first level and executes database requests management. 
- Second level is applications level and represents logical level where functional components rolling.

- The last level is user's level and from here they access application.

This architecture use optimum the hardware and software resources because the functionalities are distributed between the two servers type: application server and database server.

Regarding software, our option was to use open source software (OSS) with free sources, these products having a remarkable quality because these projects often get together distinguished programmers and because everything is public, open and anybody can come (www.opensource.org). Sources access and modified possibility is a great advantage even if very rarely somebody makes these modifications.

For added, accessed and processed database information is necessary to have a database administration system and for this site was used PostgreSQL, an objectrelational database management system (www.postgresql.org). A Relational Database Management System (RDBMS) is a complex software program (Darie et al., 2006), which purpose is to store, manage and retrieve data as quickly and reliable as possible. PostgreSQL use the SQL language - Structured Query Language, the most prevalent standardized language for database interrogation (The PostgreSQL Global Development Group, 1996-2003). There were created beginner (see figure 11), medium, advanced and master tables for tests evidence (see in figure 11 an example of the dates introducing in the beginner table using PHP language) in the e-learning database. The corresponding files (with download tests) can be put on server in a specific folder (e.g. Pdf for files.pdf, Doc for files.doc, etc.). From the downloaded page (e.g. archieved.html) the database is accessed and after that the selected test can be downloaded. All PostgreSQL features are support by graphical interface of PgAdmin III, interface who makes administration to be very easy (www.pgadmin.org).

A language that can translate the browser request, process these request, interact with software on server to satisfy the requests and to indicate to the server exactly which need to send back to user's browser is request. These languages, which work with the server and can execute these, are middleware. Such as middleware languages class was used PHP, a server-side, web scripting software. PHP is a scripting language specialized for the applications developing through code integration in the HTML document. PHP syntax derived from C, Java and Perl (www.php.net). The difference between PHP and other similar products, such as JavaScript is, that PHP is executing on the server when JavaScript is execute on the user computer.

A HTTP (Hypertext Transport Protocol) server is requested for application rolling. A good choice is Apache because of its flexibility, portability, safety and extensibility (www.apache.org).

The interfaces with users are indicating to be simply used, friendly, and attractive, without many graphical pictures or animations because they can make loading operation slower. For the forms there can be used different language as HTML (Hypertext Markup Language), CSS (Cascading Style Sheets) or JavaScript. Easy to used is HTML because this language can be edited in any text editor software 
and integrated the PHP script. The most used HTML editors are Notepad for Windows and Pico for Linux.

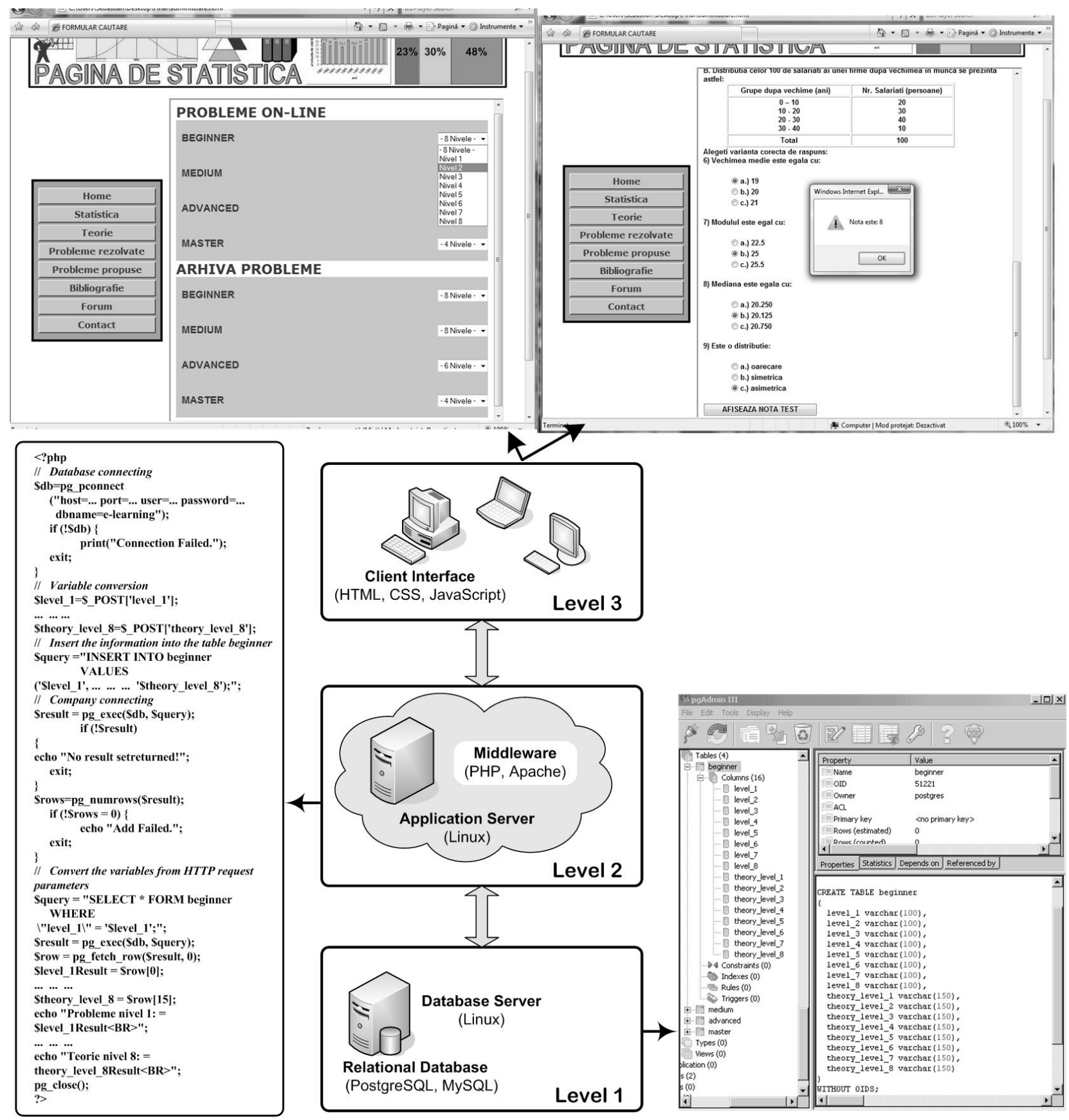

Fig. 11. A 3 levels Client-Server e-learning site architecture

In his multiple forms, e-learning offers a lot of advantages for enterprise and for employees such as (Rosu et al., b2008):

- a personalized training experience;

- low cost (e.g. travel cost for training elimination);

- access from many parts (it is necessary just a Intranet connection);

- collaboration learning based on mutual reliance and exchange of information between participants;

- total accessibility to courses and complementary materials (e-library);

- access to all resources at the same time; interactive technologies utilization; 
- learning in the proper rhythm, in a personal manner;

- better understood courses presented by means of multimedia;

- storage rooms and numerous training personnel elimination;

- the employees' ability to choose the course materials for printing and the materials for electronic format keeping; shipping low cost;

- courses dispensed in modules, each module having at the end an evaluating test at the beginning and the end of the course provide tests to see the employees' knowledge level, diversity learning mediums integration (liveliness, movies, sound), etc.

If, in the enterprise, we have many databases or many applications, the storage, protection and loosing date's problem appear. A solution is to create and to implement own Data Center (figure 12) based on multiple storage solution determine by used applications.

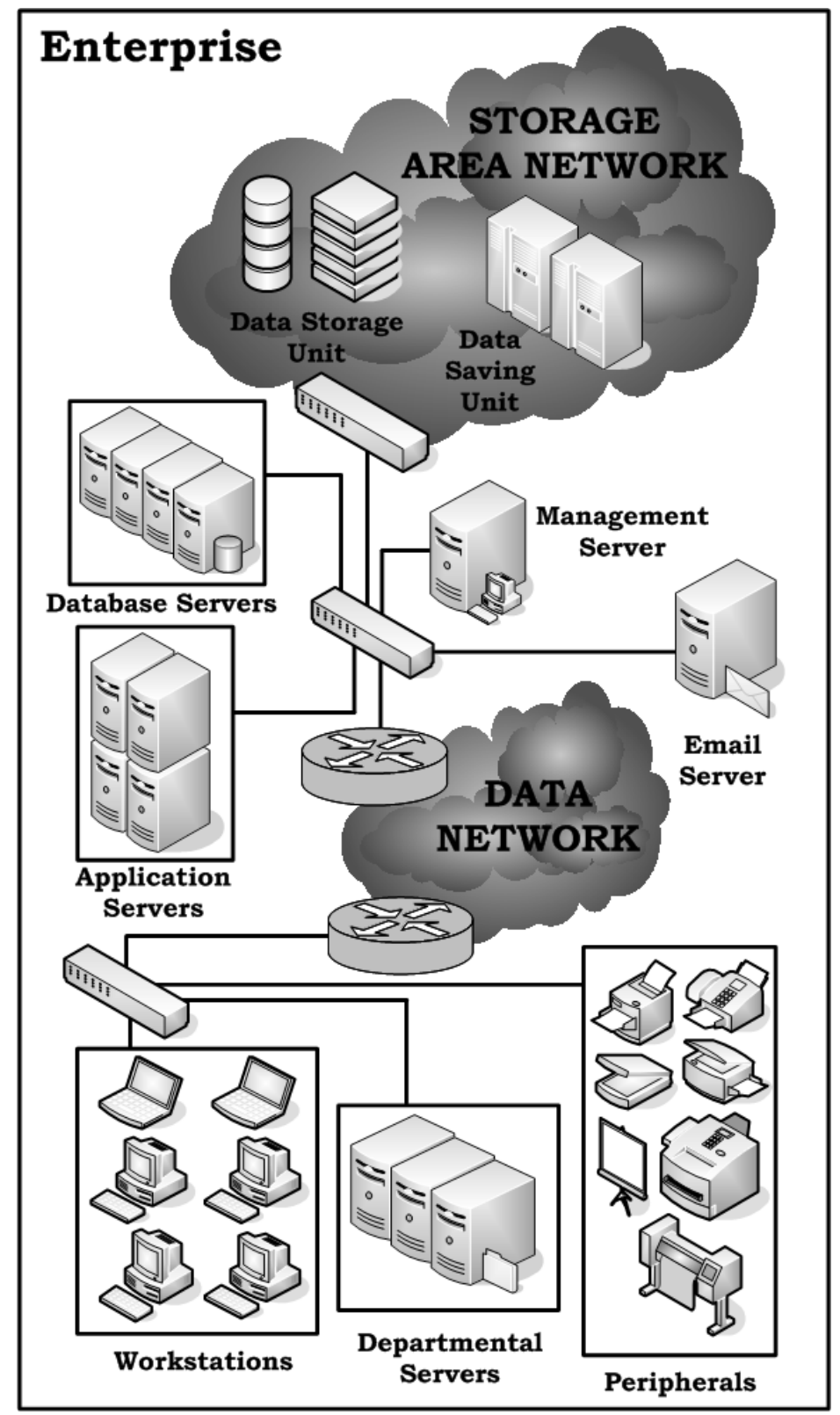

Fig. 12. Enterprise data center general architecture 
A Data Center (see figure 12) (Rosu et al., 2009) admit all authorized user access, dates backup and restore, and dates procurements in complete destruction case. The storage data system will be protecting with dates loosing or partial/total destruction default. In the modern data centers resources are used efficient by all users, because accessing possibilities, multiple storage system emerging in different locations. Every department's employees can find minimum packages of data, necessary to start their part. To have good result from the beginning is good for each department to have a database administrator who make permanent database update, accordind to the enterprise inside or outside dates provided.

\section{Conclusion}

In this paper we present a collaborative work at the enterprise level through virtual teams for project development and a network for large enterprise geographic dispersed was designed as support for VPNs possible structures to create a virtual enterprise. Also, the VPNs solutions was presented according to enterprise network territorial expansion: local, metropolitan (county) and national (international).

The concept of collaborative work has emerged both as an effect of globalization and as a prospective tool for enabling this new business approach. The opportunities and limitations presented by collaborative design, however, are not well understood, and the actual gains of applying collaborative design are not clear. Cooperative processes are not the automatic results of implementing collaborative, real-time communication technologies, but the result of a carefully designed and systematically maintained virtual team development plan. A survey of recent collaborative design research shows a focus on developing tools to facilitate communication of ideas and information within collaborative design teams.

In addition, it was presented a work method for a three levels e-learning site realization at the enterprise level according to OMT methodology and using open source software. The scope of this study (realized in the PREMINV Research Center, University „Politehnica” of Bucharest in the ORGVIRT project - CEEX PC-D03PT00-1034 between 2006-2008) was to see if these products can represent an alternative solutions to commercial software for client-server applications development at the enterprise level.

Must be known than open source products aren't in all cases better then commercial software. There is software Open Source who excels any commercial alternative as performance, but there is inferior software to comparative commercial software. Open source software with free sources is a good option these products having a remarkable quality because of fact then often these projects get together distinguished programmers and everything is public, open and anybody can come. Very well it's than management team to know from the beginning what are the costs (human and material) and how much will be during the project. Generally, to realize and to implement a database project are requisite a period within few months and few years. Certainly, in the actual market the price makes the rules. Enterprise software solution is influence by shrift, medium or long-term calculation (Rosu et al., a2008). Decision can be take consequence a comparative analysis. 


\section{References}

Darie, C.; Balanescu, E. \& Bucica, M. (2006). Beginning PHP and PostgreSQL ECommerce: From Novice to Professional, published by Apress, December, ISBN 1-59059-648-x

Dragoi, G.; Cotet, C.; Rosu, L. \& Rosu, S. M. (2006). Role of the virtual networks in the virtual enterprise. Strojniški Vestnik - Journal of Mechanical Engineering, Vol. 52, No. 7-8, pp. 526-531, ISSN 0039-2480

Dragoi, G.; Rosu, S.M.; Rosu, L.; Cotet, C.E. \& Dragomirescu, C.G. (2007). Collaborative Design System to Improve Virtual Development of Mechatronic Products, Chapter 20 in DAAAM International Scientific Book 2007, B. Katalinic (Ed.), published by DAAAM International, Vienna, Austria, pp. 213228, ISSN 1726-9687, ISBN 3-901509-60-7

Rosu, S.M.; Dragoi, G.; Guran, M. \& Roşu, L. (a2008). Relational Database System Support for Products Development Process at the Enterprise Level Using Open Source Software, Chapter 58 in DAAAM International Scientific Book 2008, B. Katalinic (Ed.), published by DAAAM International, Vienna, Austria, pp. 685-696, ISSN 1726-9687, ISBN 978-3-901509-66-7

Rosu, S.M.; Rosu, L. \& Guran, M. (b2008). Enterprise e-Learning Sites Development Using OMT Methodology, Annals of DAAAM for 2008 \& Proceedings of the $19^{\text {th }}$ International DAAAM Symposium, October $22^{\text {th }}-25^{\text {th }}$, Katalinic, B. (Ed.), published by DAAAM International, Vienna, Austria, pp. 1193-1194, ISSN 1726-9679, ISBN 3-901509-58-5

Rosu, S.M.; Dragoi, G. \& Guran, M. (2009). A Knowledge Management Scenario to Support Knowledge Applications Development in Small and Medium Enterprises. Advances in Electrical and Computer Engineering, Vol. 9 (16), No. 1/2009, pp. 8-15, ISSN 1582-7445 (print), ISSN 1844-7600 (on-line)

Shakya, A.; Takeda, H. \& Wuwongse, V. (2008). StYLiD: Social Information Sharing with Free Creation of Structured Linked Data, Proceedings of the Workshop on Social Web and Knowledge Management - SWKM 2008, April $22^{\text {th }}$, Beijing, China, ISBN 978-1-60558-085-2

Thuraisingham, B.M. (2003). Web Data Mining and Applications in Business Intelligence and Counter-Terrorism, published by CRC Press LLC, Boca Raton, Florida, ISBN 0-8493-1460-7

Ward, J. \& Peppard., J. (2002). Strategic planning for information systems, published by John Wiley \& Sons inc., West Sussex, England, ISBN 0-470-84147-8 *** http://www.cisco.com, Accesed on: 2009-02-07

*** http://www.php.net, Accesed on: 2009-03-23

*** http://www.apache.org, Accesed on: 2009-03-23

*** http://www.opensource.org, Accesed on: 2009-03-25

*** http://www.postgresql.org, Accesed on: 2009-03-25

*** http://www.pgadmin.org, Accesed on: 2009-03-27

*** The PostgreSQL Global Development Group (1996-2003). PostgreSQL 7.4.2 Documentation, Download from: http://www.postgresql.org/docs/manuals 